\title{
Ethical and practical considerations for mitigating risks to sexual partners during analytical treatment interruptions in HIV cure-related research
}

\author{
Karine Dubé ${ }^{1} \oplus$, John Kanazawa ${ }^{1}$, Lynda Dee ${ }^{2,3,4}$, Jeff Taylor ${ }^{3,4,5,6}$, \\ Danielle M. Campbell ${ }^{3,4,7}$, Brandon Brown ${ }^{8} \odot$, Mallory 0. Johnson', \\ Parya Saberi ${ }^{9}$, John A. Sauceda ${ }^{9}$, Jeremy Sugarman ${ }^{10}$ and \\ Michael J. Peluso ${ }^{11}$
}

${ }^{1}$ Gillings School of Global Public Health, University of North Carolina at Chapel Hill, Chapel Hill, NC, USA; ${ }^{2}$ AIDS Action Baltimore, Baltimore, MD, USA; ${ }^{3}$ Delaney AIDS Research Enterprise (DARE) CAB, San Francisco, CA, USA; ${ }^{4}$ Martin Delaney Collaboratory Community Advisory Board, Nationwide, Baltimore, MD, USA; ${ }^{5} \mathrm{HIV}+$ Aging Research Project - Palm Springs (HARP-PS), Palm Springs, CA, USA; ${ }^{6}$ AntiViral Research Center (AVRC) CAB, University of California San Diego, San Diego, CA, USA; ${ }^{7}$ Charles R. Drew University of Medicine and Science, Los Angeles, CA, USA; ${ }^{8}$ Department of Social Medicine, Population and Public Health, Center for Healthy Communities, University of California, Riverside, Riverside, CA, USA; ${ }^{9}$ Center for AIDS Prevention Studies (CAPS), Division of Prevention Sciences, UCSF, San Francisco, CA, USA; ${ }^{10}$ Johns Hopkins Berman Institute for Bioethics, Baltimore, MD, USA; ${ }^{11}$ Division of HIV, Infectious Diseases and Global Medicine, University of California, San Francisco, CA, USA

Background: Analytical treatment interruptions (ATIS) in HIV cure-related research can result in trial participants becoming viremic with HIV, placing HIV-negative sexual partners at elevated risk of acquiring HIV.

Objective: Our study aimed to generate ethical and practical considerations for designing and implementing appropriate risk mitigation strategies to reduce unintended HIV transmission events during ATIs.

Methods: We conducted 21 in-depth interviews with five types of informants: bioethicists, community members, biomedical HIV cure researchers, socio-behavioral scientists/epidemiologists, and HIV care providers. We used conventional content analysis to analyze the data and generate considerations.

Results: Key findings include: 1) Ethical permissibility of ATI trials depends on due diligence and informed consent to mitigate risks to participants and their sexual partners; 2) Participants should receive adequate support and/or counseling if they choose to disclose ATI participation to their partners; 3) Measures to protect sexual partners of trial participants from HIV transmission during ATIs should include referral to and/or provision of pre-exposure prophylaxis, as well as other available means of preventing HIV transmission; 4) There is uncertainty regarding the appropriate management of emerging sexually transmitted infections during ATI trials and possible protection measures for multiple and/or anonymous partners of ATI trial participants.

Conclusion: While there is no way to completely eliminate the risk of HIV transmission to sexual partners during ATIs, HIV cure trialists and sponsors should consider the ethical concerns related to the sexual partners of ATI participants. Doing so is essential to ensuring the welfare of participants, their partners and the trustworthiness of research.

KEYWORDS: HIV, HIV cure research, analytical treatment interruptions, people living with HIV, partner protection, risk mitigation

Correspondence to: Karine Dubé, DrPH, MPhil (Oxon), UNC Gillings School of Global Public Health, 4108 McGavran-Greenberg Hall, Chapel Hill, NC 27516, USA. Email: karine_dube@med.unc.edu

\section{Background}

Many HIV-negative individuals now rely on their sexual partner(s)' adherence to antiretroviral therapy (ART) and undetectable status to prevent acquiring 
HIV through sex. ${ }^{1}$ However, in HIV cure-related studies, virally suppressed people living with HIV (PLWH) may be asked to participate in an analytical treatment interruption (ATI) - pausing HIV medications, and undergoing careful monitoring of plasma HIV RNA and CD4+ T cell counts. ${ }^{2-4}$ During the ATI, participants' undetectable status may be temporarily lost as viremia is expected to return in most cases, often unpredictably. This leads to HIV-negative sexual partners being at elevated risk of acquiring HIV since the participant's HIV may no longer be undetectable. $^{3}$ In scenarios where both participants and partners are living with HIV, there is a risk of superinfection ${ }^{5}$ (i.e., a person with established infection acquires a second strain of HIV), although this occurrence is very rare. Clinical risks to participants and their sexual partners are heightened the longer an ATI lasts. ${ }^{6}$

Sexual partners of participants undergoing an ATI are usually not considered research participants. ${ }^{6,7}$ Ethically and practically, it is unclear what partner protection measures are owed to them. ${ }^{6,7}$ Further, HIV prevention and behavioral risk reduction strategies (e.g., promoting use of condoms) are considered to fall outside of the scope of HIV cure clinical research. ${ }^{3,8}$ To date, there have been two documented unintended HIV transmissions in the context of ATIs. ${ }^{9-12}$ These cases highlight the importance of planning partner protection measures in HIV cure-related studies involving ATIs.

Scholarly attention has recently focused on the ethical and philosophical considerations related to transmission risks and protections for sexual partners of ATI study participants. ${ }^{6,8,11,13-15}$ Subsequently, a team at the University of California, San Francisco proposed a possible risk mitigation package to protect study participants and their sexual partners in HIV cure-related studies involving ATIs. ${ }^{3}$ However, to date, there has been no empirical research to identify stakeholders' perceptions related to risk mitigation strategies and protection measures in the context of HIV cure-related ATI trials.

As ATI trials are scaled up, ${ }^{16}$ there is a unique window of opportunity to generate actionable considerations to design and implement risk mitigation strategies aimed at reducing unintended HIV transmission events during ATIs. This study used in-depth key informant interview data from U.S.-based stakeholders to identify perceptions of strategies to reduce unintended HIV transmission risks during ATIs, including opportunities and challenges to operationalize these strategies. This study elicited perceptions of bioethicists, biomedical HIV care providers, community members, socio-behavioral scientists/epidemiologists, and HIV care providers on proposed participant and partner-level protection strategies. ${ }^{3}$

\section{Methods Study setting and participants}

Using a purposive, non-probabilistic sampling technique, we conducted 21 interviews with five types of key informants: 1) bioethicists; 2) community members, (e.g., people living with HIV and their advocates affiliated with HIV cure research networks such as the Martin Delaney Collaboratories Towards an HIV Cure and the AIDS Clinical Trials Group); 3) biomedical HIV cure researchers; 4) socio-behavioral scientists/ epidemiologists; and 5) HIV care providers. These groups were chosen because they represent key stakeholders in the search towards an HIV cure, and their buy-in will be critical to move research efforts forward. An External Advisory Group (B.B., J.A.S. and J.S.) proposed and reviewed an initial list of potential informants and provided suggestions for additional informants. Prospective informants were from academic institutions, community advisory boards (CABs), community-based organizations (CBOs), government, non-governmental organizations (NGOs), and the pharmaceutical industry. Participants were recruited based on their experience with the topics of HIV cure-related research, ATIs, risk mitigation strategies for sexual partners, and/or HIV prevention concepts. Our goal was to obtain different viewpoints on risk mitigation measures for sexual partners during ATIs employed in HIV cure-related research. Due to the exploratory nature of this topic, we used in-depth interviews to elicit rich, thoughtful, and nuanced considerations for a topic about which little was previously known. ${ }^{17}$

\section{Participant recruitment}

The study's principal investigator (K.D.) sent formal email invitations to potential key informants asking them to participate. Email communications indicated the purpose of the study and appended the institutional review board (IRB)-approved informed consent form, demographic sheet, and interview guide. The initial list of potential informants contained 30 individuals, 21 of whom agreed to be interviewed ( $70 \%$ response rate). Only those who responded to our initial invitation were considered for an interview. Upon confirmation of the day and time of the interview, the principal investigator (K.D.) sent a Health Insurance Portability and Accountability Act (HIPAA)compliant virtual conferencing weblink to each informant. Participant identification numbers were assigned sequentially on the day of the interview. 
Table 1. IRB-Approved Interview guide: Ethical and practical considerations for mitigating risks to sexual partners during ATIs in HIV cure-related research.

Introduction

- First, thank you so much for your time.

- Can you please describe your involvement in HIV-related research?

Considerations for ATI Participants

- What should research teams do during the informed consent process of ATI study participants?

- Do you think ATI study participants should disclose their HIV status to their sexual partners?

- What types of support should be provided to participants who want to disclose their status to their partner(s)?

- Do you think ATI study participants should disclose their participation to their sexual partners? What if their partner is HIV-negative?

- What should research teams do if study participants do not want to inform their sexual partners? What if their partner is HIV-negative?

- What materials should be developed to help ATI study participants navigate the ATI process?

- What should research teams do if ATI study participants have new sexually transmitted infections (STIs) (STIs may indicate unprotected sexual intercourse during ATIS)?

Considerations for Sexual Partners of ATI Study Participants

- Do you think research teams should refer sexual partners of ATI study participants to receive PrEP (particularly if they are HIV-negative)?

- Do you think partners should be asked to come to the study site to receive information?

- Do you think partners should be asked to complete an informed consent process?

- What should research teams do in case of multiple and/or anonymous partners?

- What materials should be developed to support sexual partners of ATI study participants?

\section{Data collection}

Interviewers (K.D. and J.K.) conducted all interviews in English using a virtual conferencing platform, following the IRB-approved interview guide (Table 1). Interviews took between 30-60 minutes. Following each interview, interviewers wrote detailed field notes and updated study management tools, such as demographic logs and participant master lists. Community representatives (from CABs, CBOs or NGOs) were compensated with an electronic US \$20 e-gift card. Informants from academic institutions, industry, and government did not receive compensation.

\section{Data analysis}

Interviews were professionally transcribed. One member of the research team (J.K.) reviewed all transcripts for accuracy and completeness against the audio recordings. Audio files were destroyed once transcripts were cross-checked for accuracy, quality, and authenticity. Due to the exploratory nature of the research topic, we used conventional content analysis involving inductive reasoning to analyze these qualitative data. ${ }^{17} \mathrm{We}$ reduced the interview data and generated salient considerations based on critical inputs from informants.

One member of the research team (J.K.) compiled all de-identified text responses to queries into one master document for manual coding. To realize the potential of the dataset, we analyzed data by question blocks as well as by informant types. After reviewing responses to each question, we extracted salient quotes and ascribed codes or themes. Two members of the research team (K.D. and J.K.) double-coded the data and organized text units into emergent themes. Our codebook was inductive and contained code names, brief descriptions, and examples for each code or theme. In the process of coding, we expanded and collapsed codes and themes as needed. The coding team resolved discrepancies by discussion and consensus during virtual meetings. After the coding process was completed, we summarized patterns in the codes and prepared narrative summaries. We also derived possible implications of the findings for future practice.

\section{Ethics statement}

The University of North Carolina at Chapel Hill (UNC-CH) IRB approved this study (study \#19-0522) with oral consent.

\section{Results}

Interview participants included 11 cisgender men and 10 cisgender women. Of these, 13 were White/Caucasian, 7 were Black/African-American, and 1 was White/ Hispanic (Table 2). We interviewed 2 bioethicists, 9 community members, 6 biomedical HIV cure researchers, 3 socio-behavioral scientists/epidemiologists, and 1 HIV care provider not involved in HIV cure-related research. Interview participants worked in the field of HIV for a mean of 20.4 years ( $\mathrm{SD}=9.1$ years), and in HIV cure-related research for a mean of 6.8 years $(\mathrm{SD}=$ 6.1 years). We present considerations for ATI trial participants and their sexual partners in turn.

\section{Considerations for ATI trial participants}

Considerations include: 1) the informed consent process, 2) disclosure of ATIs, 3) non-disclosure of ATIs and risk of HIV transmission, 4) materials and resources for participants, and 5) approach to emerging sexually transmitted infections (STIs). 
Table 2. Demographic characteristics of key informant interview participants.

\begin{tabular}{llll}
\hline Participant Number & Sex & Race/Ethnicity & Informant Type \\
\hline 01 & Male & White/Caucasian & Biomedical researcher* \\
02 & Male & White/Caucasian & Biomedical researcher \\
03 & Male & White/Caucasian & Bioethicist \\
04 & Female & Black/African American & Community member \\
05 & Female & Black/African American & Socio-behavioral researcher/epidemiologist \\
06 & Male & White/Caucasian & Community member \\
07 & Female & Black/African American & Community member \\
08 & Female & White/Caucasian & Biomedical researcher \\
09 & Male & Black/African American & Community member \\
10 & Male & White/Caucasian & Biomedical researcher \\
11 & Male & White/Caucasian & Biomedical researcher* \\
12 & Male & White/Caucasian & Community member \\
13 & Male & White/Hispanic & Community member \\
14 & Male & White/Caucasian & Socio-behavioral researcher/epidemiologist \\
15 & Female & Black/African American & Community member \\
16 & Male & Black/African American & Community member \\
17 & Female & White/Caucasian & Community member \\
18 & Female & White/Caucasian & HIV care provider \\
19 & Female & White/Caucasian & Bioethicist \\
20 & Female & White/Caucasian & Biomedical researcher \\
21 & Female & Black/African American & Socio-behavioral researcher/epidemiologist \\
\hline
\end{tabular}

*Biomedical researchers with active HIV care responsibilities. For this study, they were interviewed as biomedical researchers.

\section{Informed consent process}

We asked informants to describe considerations for the informed consent process for enrolling ATI participants in research. Most mentioned the need for simple and clear information, particularly around potential clinical risks for participants and their sexual partners. Bioethicists described the need to be explicit about both clinical risks and behavioral expectations of ATIs as well as an assessment of understanding.

\footnotetext{
During the informed consent process, of course, all risks to the individual and to their partners should be disclosed. Comprehension should be assessed. - Bioethicist (\#03)
}

In turn, community members reported that there should be an evaluation of a participant's readiness to join a trial. They stressed the importance of making informed consent language as simple as possible. Some suggested summarizing key aspects of ATI trials or using videos to further facilitate understanding. They also suggested providing ample time to participants to discuss with their partner(s) and HIV care providers. Importantly, community members emphasized the need for clear language around the experimental nature of the trial and the potential HIV transmission risk to sexual partners.

[Y]ou need to explain to them exactly what an ATI is, you know, what the potential implications are. Obviously, you know, if they're sexually active ... the potential transmission of virus, once the virus levels get up to a certain level. - Community member (\#06)

Biomedical HIV cure researchers insisted on the need to inform participants about implications of the ATIs from the very beginning of clinical trials, including the recruitment process (\#01). They also emphasized the need to make clear to potential trial participants the scientific rationale for the ATI.

[W] e need to reassure people that there's a rationale for doing the ATI. We need to tell them that our protocol has set as a minimum that your immune system must have started, must have increased in its anti-HIV immune response by this much to this level before we will ask you to do an ATI, that we have given them some assurance that we are cognizant of the risks of them going off medication. - Biomedical HIV cure researcher (\#OI)

Like other informant types, biomedical HIV cure researchers identified the need to clearly inform ATI trial participants about the clinical risks, including the risk of viral rebound and the possibility of transmitting HIV to sexual partners.

I think you have to make them totally aware of all of the risks and the theoretical as well as the real ones... So, the real risk that is out there, that we have no data... is how many episodes of unprotected sex have we had with somebody who's susceptible during a period of viremia to see what the risk is. -Biomedical HIV cure researcher (\#I0)

One biomedical researcher (\#20) emphasized the informed consent process should involve discussion to guide an ATI trial candidate around their decision to participate.

[O]ne of the most crucial things that an investigator can do in an informed consent process for these studies is their best to facilitate participant understanding of all of the potential risks, the potential benefits, the potential alternatives .... But in a way that is a dialogue ... I do aspire to incorporate [a] shared decision-making philosophy and approach, particularly into these studies. - Biomedical HIV cure researcher (\#20)

Socio-behavioral scientists stressed the importance of genuine community involvement around the design and development of informed consent materials. In turn, the HIV care provider (\#18) emphasized the 
need to inform ATI trial participants about the risk of HIV transmission to partners and relevant protection measures.

Overall, informants described the need for clear language around ATI-related risks, including the risk for HIV transmission to sexual partners. They also proposed ways to enrich the informed consent process, such as through videos and companion documents. There should also be robust community engagement in determining the best strategies to approach the informed consent process in individual ATI studies.

\section{Disclosure of ATI study participation to sexual partners}

The topic of disclosing study participation to sexual partners generated a range of responses. While most informants believed disclosure of ATI research participation should not be required, biomedical HIV cure researchers believed disclosure should be strongly encouraged. Community members explained that there should be sensitivity given to relationship dynamics, as well as to underlying social, psychological, and cultural issues around disclosure.

Community members stated that, in certain circumstances, ATI trial participants should be encouraged to disclose, such as in the cases of HIV sero-different relationships, unprotected sexual intercourse with HIVnegative partners, and/or when partners rely on $\mathrm{U}=\mathrm{U}$ as their HIV prevention method. Community members felt that if ATI trial participants wished to disclose their research participation to their partners, adequate support should be provided to them.

\begin{abstract}
If they're in a serodifferent relationship and the partner is not on PrEP, then yes, I think they should disclose it, because $U=U$, essentially. If they're in a situation where they've been having unprotected sexual intercourse or condomless sex ... that could result in transmission of HIV, and basically ... treatment as prevention has been what they've used as their sole method, they're not using any barrier-type prophylactics andlor the partner's on PrEP, then if it's a partner, like a serious partner, I think they should disclose, yes.
\end{abstract}

- Community member (\#04)

One biomedical researcher (\#02) stated that participants should absolutely disclose their participation to sexual partners because transmission to an uninfected partner is one of the greatest risks of HIV cure-related research. Early-phase trials will likely be less efficacious than later-phase trials and, therefore, result in participants having unpredictable viral rebounds.

Absolutely. Absolutely. Because ... the greatest risk of these cure trials with ATIs is transmission to an uninfected partner. To me, that is the greatest risk, and it would be frankly tragic because early on, I don't know that these concepts will work... We may get low efficacy rates, which means a lot of people will be viremic and able to transmit. So therefore, it's really, really important that we are super careful and everybody's aware of what we're doing. - Biomedical HIV cure researcher (\#02)

Socio-behavioral scientists focused on issues surrounding sensitivity to participants' unique circumstances. A suggestion was made to help participants clarify how the ATI trial will affect everyone around them, including their sexual partners.

[Y]ou have to be really both sensitive to the person, but also this is where clearly making sure that they understand kind of what this trial is ... and then how that can then affect the health of their partners, their families, their community, whatever it is that resonates with them. - Socio-behavioral scientist (\#2I)

The HIV care provider (\#18) did not believe that ATI research disclosure should be required as part of the protocol. In conversation with partners, value and meaning should be ascribed to the act of HIV treatment interruption to help advance the science of HIV cure.

\footnotetext{
I don't think they have to, but it probably would be helpful for them to explain to their partners that they're doing this as part of a science project to cure HIV. It seems much more meaningful, rather than just to tell your sexual partner: "Hey, I decided to go off my meds, let's go have sex." I would think that if it was me, I would explain all this, but I don't think it needs to be obligatory or required. - HIV care provider (\#|8)
}

The general perception amongst our informants was that disclosure of ATI research participation should be strongly encouraged, but not be mandated. Support should be offered to participants and researchers should remain sensitive to relationship dynamics.

\section{Considerations for Non-ATI disclosure and risk of HIV transmission}

We asked respondents to consider situations when ATI research participation had not been disclosed to sexual partners and where there may be a risk of HIV transmission. Most informants appreciated the complexity of such situations. A bioethicist (\#03) discussed the participants' non-enforceable moral duty to disclose their possible viremic status.

Community members emphasized the need for regular counseling on the risks of HIV transmission to sexual partners during ATIs, as well as ongoing dialogue and education during the trial. Once again, community members believed attention should also be paid to the complexity of relationship dynamics and of social and sexual networks.

[E]ach case I think is gonna be different ... Like all of our decisions are influenced by the thoughts, ideas, beliefs, and behaviors of those around us, also the social norms in people's families, you know, social networks... So, you know, there needs to be a persistent kind of communication and engagement with the participant around, you know, what their participation means in the context of their relationships, and vice versa.

- Community member (\#I6) 
Further, community members suggested mental health support be available to ATI trial participants throughout the study.

[M]ental health clinician, social worker, you know, psychologist, what have you, to kind of continue to have these conversations with the participants, throughout their participation in the study.

- Community member \#16

Biomedical researchers discussed the need for transparency between research teams and ATI trial participants, particularly in early-phase trials.

That means that the scientists, the physicians, the participants, their partners, you have to be transparent. And the reason it's so important be transparent is because of those ATIs. If somebody is undergoing [an] ATI, the partner must know that this is a clinical trial, this is a temporary situation, but they must understand the risk.

- Biomedical HIV cure researcher (\#02)

A biomedical researcher (\#11) explained that in such thorny situations of non-disclosure, additional guidance should be sought either from the IRB or the CAB.

I wish that there was an easy answer to that question. That is tough ... Ideally, part of the issue becomes once you're aware of the issue, we try to get a sense of what level of risk might be associated with the ATI, and so we're usually aware of issues like that ... I think that is certainly a situation that needs more guidance ... it would be the type of thing that we would seek advice from both the IRB and our community advisory board with whom we work very closely. - Biomedical HIV cure researcher (\#II)

Another biomedical researcher (\#20) described how each trial participant's situation is unique. Researchers should get to know each participant and address complex scenarios on a case-by-case basis.

[E]very situation is really quite individual. So it's my job, as investigator, to get to know that participant, or potential participant... and understand what he, she, or they are capable of in terms of dynamics in their relationship. I try to not misunderstand the dynamics of a relationship, I want to engage and have a trusting conversation with the potential participant about it, and be sure that they understand the risks of the study... during a treatment interruption a majority of participants will have a detectable viral load, and an increased risk of transmission to others. They may also have, depending on the status of their partner, an increased risk of super infection or of acquisition from their partner or partners.

- Biomedical HIV cure researcher (\#20)

In turn, the HIV care provider (\#18) contended that by law in some U.S. states, participants must disclose their HIV status if they are viremic and having unprotected sex. This could be framed in a positive light given that some states are beginning to modernize their laws; however, this may be dependent on the specific state.

[B] ut by law they have to disclose their status if they are viremic and they have to wear a condom, too, if they're viremic. I think unfortunately you might have to say something to those along those lines without scaring away your study participants ... One way to make it a positive thing is to explain that [a U.S. state] actually does have these modern laws where we understand the science here, when you're undetectable you can't transmit it, we can explain all that... There's a way to spin it I think to make it seem positive but then make sure that the patient understands that they will have to disclose during this, at the time of this research project.

\section{- HIV care provider (\#I8)}

Informants stated the need for regular mental health counseling and support, dialogue, and education; and attention to the complex relationship dynamics and social/sexual networks. Additional guidance should be sought from the IRB or the CAB as needed, and situations should be handled on a case-by-case basis. Participants should also be made aware of the laws around HIV transmission in their state or area.

\section{Materials and resources for ATI trial participants}

We asked informants to provide suggestions on materials and resources to help participants navigate the ATI period. A bioethicist (\#19) recommended innovative approaches such as mobile health technologies, text messaging, or a trusted advisor who could regularly check in on ATI participants.

\footnotetext{
I know people have used a lot of stuff with mobile health and text messaging reminders and things like that, and you know, maybe other things that you could try could be like a buddy system, you know, where you have a person who check in who is like a trusted, confidential sort of adviser type person, like an ombudsman-type person, you know? - Bioethicist (\#19)
}

A community member (\#04) uniquely commented on the need to balance health and ethics with sex positivity to ensure ATI trial participants were not portrayed as vectors of virus transmission.

[W] want to make sure that we balance health and ethics with sex positivity. I think a lot of times there's too much pressure ... to try to conduct themselves so they're not "a vector of infection."

... Every human being, regardless of their serostatus, has a right to a fulfilling and positive autonomous sex life, which can include condomless sex. - Community member (\#04)

Community members highlighted the need for clear materials around ATIs, the associated transmission risks, and participants' trial responsibilities. Possible ways to convey complex information included documents to accompany the informed consent process, videos, presentations, surveys, and fact sheets of frequently asked questions. These materials could also be adapted to explain ATIs to HIV care providers or for community engagement efforts. Ultimately, community members stated that materials should help empower trial participants to weigh possible trade-offs of going through the ATI with disclosing their HIV status and/ or research participation. 
Biomedical researchers similarly discussed the need for additional materials to support the informed consent process. One biomedical researcher (\#08) working at an African research site described the importance of relying on visual language, workshops to encourage dialogue around difficult topics, and even role-playing exercises.

In turn, socio-behavioral scientists stressed the importance of having mental health resources available to participants, particularly if they have difficulty disclosing their HIV status or research participation to partners.

I always make sure that I'm able to find referrals for free mental health counseling for anything about the process ... if they're struggling with disclosing to partners. - Socio-behavioral scientist (\#05)

Socio-behavioral scientists further highlighted the need to pay attention to literacy and numeracy levels of stakeholders. There could also be websites developed where participants could find more information about ATIs and third-party partner risks. Another suggestion was to connect participants to other willing ATI trial participants for support. An HIV care provider (\#18) suggested that "handouts or probably ... something on a paper is recommended" (\#18). Overall, informants offered multiple suggestions to help simplify partner protection messages before and during the ATI.

\section{Considerations for ATI trial participants who develop STIS}

STIs may be indicative of unprotected sexual intercourse. Informants diverged in opinions around whether ATI participants should be allowed to stay in the trial if they develop STIs.

A bioethicist (\#19) argued that incident STIs should be approached from public health and clinician standpoints. Research teams should ensure proper counseling, contact tracing and referral systems are in place before the trial because biomedical HIV cure researchers are not contact tracers.

I would kind of go back again to sort of this combination of what would a clinician do, and what would a public health person do? ... The researcher's responsibility I think would be to make sure that those mechanisms were in place for follow-up... You're probably going to have a risk reduction counselor available, so somebody who can do counseling, somebody who could do contact tracing would be important because the contact tracing would address both the STI and the HIV risk, you know? ... HIV cure researchers are not contact tracers.

- Bioethicist (\#19)

A community member (\#17) described how STI prevention counseling conversations may need to be frequently revisited during a trial, because a participant's circumstances and partnership dynamics may change. Therefore, it is important to constantly revisit the risk reduction plan during a trial. The importance of understanding a participant's sexual network was highlighted by another community member (\#12). This informant described the impossibility of being able to control every scenario, even within the context of controlled clinical trials.

[Try] to understand their partner network; is it their regular partner, is it a different partner? ... Ideally, you'd want to get their partner or partners into care, and that can be very complicated if they're not in a steady relationship or even if they are ... in a clinical trial you can't control everything as much as you're designing a controlled study. - Community member (\#I2)

One community member (\#13) reported that participants with repeat STIs may need to be discontinued from the ATI trial. Other community members encouraged research teams to consult with HIV/STI prevention experts before and during the ATI trial. Special circumstances, such as intimate partner violence, would require additional support.

As emphasized by biomedical HIV cure researchers, STI test results may not reveal the complexity of each incident. There can be different types of STIs and transmission routes that further complicate analysis and prevention. STIs may also trigger viremia and affect trial outcomes.

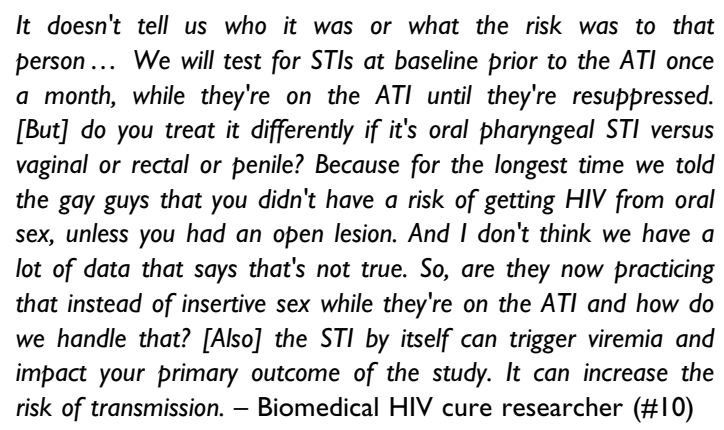

Two biomedical researchers (\#01, \#20) indicated that it would be punitive to remove participants with incident STIs from an ATI trial. They stated that, for some relationships, the norm has become PrEP use and condomless sex. Further, sexual and gender dynamics can be very complex, particularly in international contexts.

\footnotetext{
And I don't expect my study participants to use condoms any more than I expect my regular patients to use condoms. We talk about it, we fully inform, we have boatloads of condoms in our clinic to always give them away. Lube as well. But, again, it's a volitional act to use a condom ... I wouldn't take the participant off the study. I think that's punitive. I think that's not recognizing the fact that they are human beings ... And to take that person out of the study and just say you've been bad, you can't come back, number one is throwing some very good data perhaps out the window... Number two, that's real life... I still wouldn't kick the person out of the study. I would counsel them, I would let them know of the severity of their acts.
} 
- Biomedical HIV cure researcher (\#0I)

I may be one of few people who is really strongly not supportive of having incident STI be a strict restart criteria for ART. I just think that there's so much more nuance in what that seemingly new incident STI result can mean. And, particularly when I think about this in international populations, and particularly when I think about this in women in sub-Saharan Africa, I think that the dynamics at play here are even more complex.

- Biomedical HIV cure researcher (\#20)

However, another biomedical HIV cure researcher (\#02), stated that participants should be removed from the study if they present with incident STIs.

So if STIs are being acquired, it tells me that the participant is taking way too much risk, but basically harm themselves, harm the partner and harm the study, in which case you're hurting everyone in the process. And so basically, I would not enroll or basically would eliminate them from the study if they had an STI. It's really critical that we get it right. - Biomedical HIV cure researcher (\#02)

Two biomedical researchers (\#10, \#20) embraced the critical role of community engagement in determining the proper way to handle incident STIs during an ATI trial.

But it's how the community views this and getting their feedback is going to be key in how we move this forward, because it's the community we're trying to protect at this point. - Biomedical HIV cure researcher (\#I0)

In addition to following standards of care for treating STIs, a socio-behavioral scientist (\#05) suggested making information available to participants before and after they tested positive for STIs during the trial. The HIV care provider (\#18) recommended good STI counseling practices and believed it would be too draconian to disqualify a participant from a study due to an STI. However, repeated STIs may require more careful attention due to potential liability issues.

\footnotetext{
Yeah, we use that [STIs] as a surrogate for unprotected sex. ... If it's gonorrhea of the mouth, maybe they just received oral sex or they gave oral sex or whatever... but if it's like rectal gonorrhea or gonorrhea from the penis, it's going to be a little bit harder to explain... In terms of disqualifying patients... that's a little bit draconian I think to disqualify... I guess if they have repeated STIs, that might be something you want to consider is withdrawing the participant at that time because you probably could be held liable by somebody at the end of the day if you continue to keep them off their medications while they were having STIs. - HIV care provider, (\#I8)
}

In case of incident STIs during ATI trials, informants agreed on the need for prompt treatment, counseling, and contact tracing. Some believed discontinuing ATI participants from the trial would be too punitive, while others did not. Research teams may need to revisit counseling conversations frequently and pay careful attention to repeated STIs. Informants acknowledged the critical role of community engagement in determining best practices for handling STIs.

\section{Considerations for sexual partners of ATI trial participants}

Considerations for sexual partners include: 1) referral for PrEP, 2) asking partners to come to research sites, 3) asking partners to complete informed consent, 4) multiple or anonymous partners, and 5) materials and resources for partners.

\section{Referral for PrEP}

All informants were strongly in favor of PrEP referral and/or provision for partners. They also offered their own nuances to the complex PrEP provision and/or referral issue. A bioethicist (\#03) explained that partner protection often gets philosophically confusing because partners are not study participants, yet research teams still owe them protection.

\begin{abstract}
Some people have philosophically confused this and said that therefore the partner is a participant, but their [partner is] not a participant. We're not studying them, so they're not... a study subject [participant]... They're not subjects, but research in general can put people other than the subject at risk, and we owe them [partners] things as well. - Bioethicist (\#03)
\end{abstract}

Another bioethicist (\#19) reiterated the researchers' obligations to protect partners but explained the importance of taking social and scientific relationships into account as well.

[I]t depends on data on how people's relationships with their partners intersect with their research participation... Obviously there is privacy concerns for participants, but then there's this kind of third-party concern. Researchers need to care about the partners, even if the participant doesn't really want them to care about the partners, you know, there's an obligation there, right? But navigating that obligation all depends on how this social and scientific relationship is set up between [the] participant and research team.

- Bioethicist (\#|9)

Community members thought that PrEP should be offered in a proactive manner, perhaps even with funding and partnerships in place prior to the ATI trial start. They suggested a "warm hand-off" for partners and a public health approach to HIV cure research. Community members suggested studies could even take financial responsibility for PrEP provision and acknowledged the need for long-term relationships with PrEP specialists and health care providers beyond the study for partners.

[W]e've made our PrEP availability program available to partners. So the first step in that is, do they even know what PrEP is? We're happy to explain it, we're happy to refer you to somebody else who could explain it, it's up to the participant to help make that connection. If somebody is interested in starting PrEP, we're happy to enroll them in our program ... And we make sure that they're connected to a primary care provider because our sites are not the prescribing physician. They're merely the conduit... 
We have to make sure that they have a primary care doctor and then we can help them access the meds for free. We do that on purpose because we don't want the ability to be on PrEP to be solely tied to a study that will end. - Community member (\#|7)

Community members believed PrEP should be supplemented with additional HIV prevention measures. Partners should have access to the full HIV prevention toolbox of latest technologies available. This may include technologies other than PrEP, such as dapivirine vaginal rings for female partners or external and internal condoms. For community members, PrEP referrals were much more challenging for partners who were not in long-term relationships. They commented that relationship dynamics change, and partner protection processes should remain dynamic and adaptive during ATI trials. Once again, community members highlighted the need for robust community and stakeholder engagement to share knowledge about ATI trials. They also cautioned that ATI trials may add additional burdens and strains to already-struggling and under-funded PrEP programs.

Biomedical researchers described their current PrEP referral practices in ongoing ATI trials. These include referring partners to PrEP or providing PrEP directly. Biomedical researchers also described the need to followup with partners during the study and/or speak with partners directly to provide counseling if needed.

I think they should either refer them to a PrEP provider, or they should just give them the PrEP directly... So, I think this is something that really has to be carefully thought about and worked through... where does the investigator's responsibility end in terms of risk? ... The bar has been raised. $U=U$ [undectable = untransmissable] is a fact of life in the world today. It is the standard. And for us to take that standard and break it in half and purposely make someone able to transmit the virus again because of the protocol for a cure study, I think we do have some responsibility there... I think it's important that there be some follow up with that partner via the study. Biomedical HIV cure researcher (\#0I)

A biomedical researcher (\#01) described how one of their ATI studies paid for PrEP for monogamous partners. They explained, however, the situation becomes much more complex when there are multiple partners without medical insurance.

If there is not a monogamous partner and there are multiple partners with whom someone is having sex, that becomes much more difficult because PrEP has to be taken at least for a certain period of time before the sex occurs... So even though I could refer someone who perhaps doesn't have insurance in a Medicaid non-expansion state to a provider to get PrEP, and because they don't have insurance, the provider petitions, well maybe uses the initiative of End the Epidemic to access Truvada or Descovy for that person. But how are they going to pay for the labs? - Biomedical HIV cure researcher, (\#0I)

Two biomedical HIV cure researchers (\#08, \#20) working in international contexts described the need to thoughtfully consider socio-cultural dynamics in PrEP referrals. Robust community engagement around PrEP uptake for partners will be critical. There may also be special considerations for younger female participants asking older male partners to start PrEP. One suggestion was to offer HIV self-test kits to participants to empower them in discussions with their partners.

I think the uptake [of PrEP] will be exceedingly low. Firstly, because I think the [participants] will not even tell their partner... I would like to offer the participants five self-test kits or 12 self-test kits or however many self-test kits that they would like with the intention that they're going to test with their partner... So it's again empowering them to have a choice and to bring people into their circle where they still have so much stigma ... But I really think that self-testing is a wonderful tool to equip participant women with to engage their partners to test and discuss HIV. - Biomedical HIV cure researcher, (\#08)

A socio-behavioral scientist (\#05) further explained that there is a long way to go in raising awareness about PrEP availability, particularly in communities of color.

[W]ith my lens, working in communities of color, there is not nearly enough awareness about the availability of PrEP. If you're talking certain populations like LGBTQ+ [Lesbian, Gay, Bisexual, Transgender, Queer +] communities, yes, there's a decent amount of awareness. If you're not, if you're talking cisgender, heterosexual populations, if you're talking young people, people of color, and also some younger adults, the awareness is so low... Particularly now that we do have things like the "Ready, Set, PrEP" initiative and a lot of these other opportunities for low cost or no cost PrEP and what they call PrEP on demand. Because cost is not so much a barrier anymore, and awareness and availability is still [low].

- Socio-behavioral scientist, (\#05)

The HIV care provider (\#18) described how PrEP intake visits usually occur in the clinic, including obtaining a persons' medical history, drug allergies, and insurance status. The HIV care provider (\#18) stated that barriers to PrEP access should be reduced as much as possible if partners desire to take PrEP.

Overall, our informants were in strong support of PrEP referral and/or provision for partners, but offered critical nuances to this complex issue. Besides oral PrEP, there could be information and access to the entire HIV prevention toolbox (e.g., vaginal rings, condoms, etc.). PrEP referrals will be challenging in situations where there are multiple partners and/or those partners do not have medical insurance. There should also be attention paid to socio-cultural dynamics and lower PrEP awareness and access in certain communities.

\section{Asking partners to come to research sites}

We asked whether partners of ATI trial participants should be asked to come to research sites to receive additional counseling around HIV acquisition risks. Most informants thought partner visits should be 
offered as an option, but not mandatory. Only one bioethicist (\#03) believed requiring partners to come to research sites may be acceptable.

If we recruit only people with whom we can achieve more safety rather than anybody who wants to participate in it, that doesn't strike me as ethically discriminatory in a bad way... We want to keep your partner safe. Sorry. If the partner can't come in, if you don't want to disclose status to them or whatever, you can't be a participant of this research. - Bioethicist, (\#03)

By contrast, all community members indicated that partner visits should be offered as optional. Both participants and partners could both receive additional support and have their questions answered to their satisfaction. Another community member (\#13) described that if partners decide to come to research sites, they should be able to interact with staff with whom they can relate and who are sensitive to their needs.

$[S] 0 \ldots$ if they're asking someone to come to the site, they should have someone in the site they can relate to. Someone that understands if we're talking about communities of color, what is, you know, what are the dynamics and what does that mean? And, if they don't have that in the site, maybe they should partner with a community-based organization that, you know, know how to do that, right?

- Community member, (\#|3)

Another community member (\#04) explained it would create unreasonable worries to require partners to come to research sites. Some biomedical HIV cure researchers were receptive to engaging with partners at research sites, if needed. They stressed the need for transparency in implementing ATI research protocols. A socio-behavioral scientist (\#05) described the challenge of mandating partner visits during the COVID-19 pandemic and suggested using telehealth instead to counsel partners. Similarly, the HIV care provider (\#18) mentioned that it would be a good idea to bring in partners to the clinic if they are interested in receiving PrEP.

Most informants stated that partners should be given the option to attend research sites. They also described innovative ways of engaging partners, such as telehealth visits.

\section{Asking partners to complete an informed consent process}

All but one informant (biomedical HIV cure researcher, \#02) indicated that informed consent should not be required, unless partners are also explicitly in a sub-study. A bioethicist (\#19) believed that partner protection should be about building relationships, trust, and good communication.

I think that's an option. I don't think it should be required. You know it puts a burden on the partners that if they're going to be part of the study, they're contributing data to the study, they may not have that level of commitment, you know? ... I'm a little doubtful that informed consent and enrollment is going to necessarily be like better protection, I think you know, again, it's about relationships, about trust, communication, about making sure that people are, you know, in general willing to act on good faith in terms of understanding the risks and trying to mitigate the risks... And I don't necessarily think that informed consent's the best way to get to that state of affairs. - Bioethicist, (\#19)

The main reason community members were reluctant to obtain partner consent was the difficulty of determining the types of relationship(s) between participants and their partners. They expressed concern that requiring informed consent would add a level of unnecessary policing of sexual activity.

\begin{abstract}
Are we talking about serious partners, like domestic partnership, spouse, live-in? ... What if ethical non-monogamy is part of their life, or what if the person's single and they're just with whomever? "Partners" is a very loose word. It can mean someone that's your life partner, it can mean somebody that was your partner for about 30 minutes. What I don't want us to do is police the sexual activity of people living with HIV, because we don't police the people who don't have HIV. - Community member, (\#04)
\end{abstract}

Only one biomedical researcher (\#02) was adamant that informed consent should be required for partners for the sake of transparency.

Yes. They need to be aware. They need to be fully aware. Again, transparency. The partner is just as important as the participant, right? The participant is taking on additional risks, but because of that ATI, the partner is just as involved in my opinion in that trial as the participant. Really critical that they know everything, informed consent, provision of PrEP, everything. I mean, the two of them have to be apt to be completely aware of this.

- Biomedical HIV cure researcher, (\#02)

Socio-behavioral scientists (\#05, \#21) believed partners should be asked to provide informed consent only if they were also part of a sub-study and data were collected from them as well, such as in the case of partners or couples research.

Are you collecting data from the sexual partner? ... Also, just for the sake of the HIV cure research, sub-ancillary study partners, and in terms of partner support for people enrolled in these studies, or partner concerns or whatever. - Socio-behavioral scientist, (\#2I)

The HIV care provider (\#18) said that it was not necessary to obtain informed consent from partners. Informed consent forms can be unnecessarily lengthy, and mandating partner consent may send the wrong signals about the trial.

\footnotetext{
No... I think that sounds kind of shady. They're [informed consent forms] always so long and I feel like I'm signing my life away. I can't really even figure out why you would need one, but I do think bringing the partner in is a good idea, but to have them sign a document saying that they were there, they heard you, they understood the instructions... to me that seems like it's just [the institution] trying to not be sued or something.

- HIV care provider, (\#I8)
}

In sum, most informants believed it was unnecessary to obtain partner consent as part of an ATI trial. It may be 
difficult to know the type(s) of relationships or partnerships involved. Partner protection should be about building relationships, trust, and good communication. Informed consent should be required; however, if partners are part of a sub-study and expressly contribute scientific data.

\section{When ATI trial participants have multiple or anonym- ous partners}

Some informants thought that individuals with multiple or anonymous partners should be able to participate in ATI trials, while others thought they should be excluded. A bioethicist (\#19) was much more worried about anonymous partners rather than multiple partners.

\begin{abstract}
I think I'm less worried about the multiple partners than the anonymous partners, I mean I think people do have multiple partners in their lives... with anonymous partners... that's really just going to be very difficult to have any kind of responsible approach to protecting the partners... This is not to stigmatize people for the way that they conduct their private lives, it's more so how can you responsibly approach risk mitigation.
\end{abstract}

- Bioethicist, (\#19)

Community members voiced a range of opinions on the topic of multiple or anonymous partners. One community member (\#09) believed this should not be an exclusion or withdrawal criterion for ATI trials.

Um, I don't think anything different than they do with everybody else. I think the same kind of counseling about safer sex practices is important... 'cause you don't know, the people that you don't think are having a bunch of crazy sex are probably having a whole bunch of crazy sex. So, give everybody the same information (laughs).

- Community member, (\#09)

Another community member (\#06) cautioned against allowing participants with multiple or anonymous partners to continue into ATI trials as this may create undue risk of HIV transmission.

[T] hat becomes a risk to the, you know, study overall. And if you're aware that that person's doing it, I think that that should be a reason for not continuing forward with that study participant. - Community member, (\#06)

Community members described the need for robust standards of risk mitigation measures in ATI trials with all partners, harkening back to the days before "treatment as prevention" was recognized as an effective method of HIV prevention.

If they're participating in a cure trial where treatment interruption is one of the interventions, and they are fully aware of that, then the standard of care for prevention has to be deep and robust... So that it has to be about your partners, known, you know, anonymous, casual, you know, long-term, whatever that is, that you have to have some responsibility, if not disclosing, in practicing all the other safer behaviors and alternatives that you have before you outside of viral suppression. - Community member, (\#07)
Community advocates stressed that good communication may help create trial environments that allow participants to be in the driver's seat when it comes to partner protection. Participants bring different strengths and experiences with them, such as being willing to abstain or use condoms, while others may be unable to enforce partner protection measures.

I think there's a huge need for the research team to really work with the individual participant to understand their life, their sexual relationships, their sexual network... I think it is dutybound for the team to work mainly through the participant, and really let the participant be your guide into how to navigate that. $I$ think to set up a clear, "Thou shalt do $X$ or $Y$," as a research team in these settings, it is very complicated... I think that's the relationship you're trying to cultivate in any trial, particularly for an ATI study. You want participants to make sure they're making the right decisions, so I would really put the onus on the research team to create the environment that allows the participant in a trial to drive decision-making and the needs for him or her with respect to his or her partners. - Community member, (\#I2)

And so if you can tap into that altruism which will come through that informed consent process and hearing their stories, why they're in it, what their fears are, what their concerns are when they go through that informed consent and they can share where they're coming from, what their own experiences have been... Oh, so, you know, [they] go back to using condoms... and they don't get any STIs. And so, you don't have any real concerns. Or there are other people who might be sex workers who have not yet, you know, who are not always able to practice that, then they're going to have to have some sort of alternatives.

- Community member, (\#07)

Biomedical HIV cure researchers stressed that ATI trials should ideally not lead to HIV transmission events because unintended HIV transmissions may tremendously set back the field of HIV curerelated research.

And if the participant undergoes an ATI, has multiple partners, infects three people in the process... that is simply put, a disaster. We cannot have cure trials that yield additional numbers of infected people, because we're trying to discover the cure. That cannot happen. - Biomedical HIV cure researcher, (\#02)

A socio-behavioral scientist (\#05) suggested relying on ATI trial participants to be the main agents of information when it comes to protecting multiple or anonymous partners.

[Y]ou'll need them to be your agent of information with these anonymous partners. It's almost impossible to expect multiple, potentially anonymous partners to want to come into a study for HIV testing and PrEP counseling... You're probably not going to get direct access to them. That means you're probably going to have to ask... your ATI participant their comfort level with giving some information to these partners around confidential, anonymous HIV testing and PrEP counseling. - Socio-behavioral scientist, (\#05)

Another socio-behavioral scientist (\#21) contended that individuals with multiple or anonymous partners may not be the best candidates for ATI trials. This perspective was 
similarly expressed by the HIV care provider (\#18), who believed the situation would be much more complicated if discovered midway through an ongoing ATI trial.

I'm not sure that is the best candidate, if they're telling you, "I have multiple sexual partners. I have multiple anonymous partners." I'm also thinking about this may be someone who is engaging in transactional sex or sex work of some sort, or what have you and, I don't know that they will be able to implement safer sex practices needed in this type of trial... [The] risk may be too great... So, I'm not sure that I would allow someone to participate. - Socio-behavioral scientist, (\#2I)

I don't know if that's an exclusion criteria... because it doesn't seem like they would be a great study participant... What happens if you find out midway through that they have multiple partners or anonymous sex? I don't know. It's a very good question ... I try to keep the legal stuff out of it, because I find that scares people and it's not the right way to go. - HIV care provider, (\#|8)

In total, ATI trial participants with multiple or anonymous sexual partners generated a range of divergent opinions, from allowing these individuals to participate to excluding them up front. ATI trials will require robust standards of risk mitigation measures with all partners, and research teams will need to acknowledge people's lived experiences and foster a desire to protect partners, along with good communication. Unintended secondary HIV transmission events may considerably set back the field of HIV cure-related research.

\section{Materials and resources for partners}

Informants suggested multiple helpful materials and resources for partners, including information about the ATI trials and HIV/STI prevention measures. A bioethicist (\#03) explained there is complex literature on what research teams owe trial participants if they get injured, but this literature is not explicit about obligations to third-parties. In particular, ATI trials raise the ethical issue of whether research teams owe partners access to HIV treatment following HIV seroconversion any duty of care under current law and practice.

There is a whole kind of entire libraries of literature on what investigators owe study participants who get injured in their studies and it's a complex area... So how much the study team now owes the partner ART access for a year? For life? That arises more in international context. - Bioethicist, (\#03)

Community members suggested materials for partners, including slides, and frequently asked question documents. These materials should focus on what ATI trials mean for partners, as well as possible HIV prevention options. Community members explained the usefulness of employing multiple modalities to accommodate various learning styles.

It's almost $a$ bit of a flowchart; if your partner knows you're positive and knows you're virally suppressed and you're going to be in this ATI treatment, here's what they need to know. If your partner doesn't know your treatment status, but knows you're HIV positive, I'd like to think they probably already are focused on PrEP and condoms, etc. ... So, I think your materials need to be looking at helping your participants navigate those issues about HIV status, about treatment status, and about prevention needs as three categories and let the participant have and help them guide you to what they need to help to keep their partners safe.

- Community member, (\#12)

Another community member (\#04) advised leveraging current HIV prevention resources and/or existing informational platforms for partners and family members.

I think there are some things out there. I know that HIVE online has some great stuff for not just people who are positive, but for their partners and their family members. I know there are some resources out there that recognize that a person living with HIV is connected usually to a community, household unit or something, that there's people in their lives who may not be as informed because it's not their diagnosis.

- Community member, (\#04)

A biomedical researcher (\#10) recommended writing a letter to partners of ATI trial participants explaining the study and associated risks.

[W] could do basically a letter to the partner saying, "I want to be open and honest with you. I am HIV positive, and I'm going to be stopping ART at some point in the next $X$ number of months to participate in this study. There's no direct benefit for me, but it may help find a cure to help us in our search for a cure for HIV." During this period of time, we may need to have [to] use condoms consistently, or you may need to go on PrEP or both, because the PrEP piece will manage the HIV transmission, but it will not manage the STIs.

- Biomedical HIV cure researcher, (\#I0)

Another biomedical researcher (\#08) advised creating various scenarios to help ATI trial participants navigate their way through meaningful dialogues around risk mitigation measures with partners.

Just really play out the scenarios, but let them take the leading
role to tell us what the risk is to them and explore what is of
value to you, what do you really want. I think that kind of
truthful dialogue is invaluable for them to tell us and inform us
and for us to really listen to what those key challenges are
instead of developing that in a vacuum without participants
telling us. - Biomedical HIV cure researcher, (\#08)

In turn, socio-behavioral scientists suggested providing clear information about the study and about HIV/STI testing. Recommendations were made to explore the use of multi-media, vignettes, scripts, and text messages. Further, a socio-behavioral scientist (\#21) emphasized the need to conduct formative work to understand acceptable communication strategies around risk mitigation in the context of ATIs.

[M]aybe part of that preliminary or formative work is around understanding what some of the best strategies may be for different people, and that it may need to be a combination of strategies.

- Socio-behavioral scientist, (\#2I) 
Likewise, the HIV care provider (\#18) suggested providing handouts with information about risk and risk mitigations strategies. Together, informants provided a wealth of suggestions for ways to communicate risks and risk mitigation strategies to sexual partners of ATI trial participants. Informants advised conducting additional formative work to design optimal communication strategies for risk mitigation.

\section{Discussion}

Our findings point to possible risk mitigation strategies to protect ATI trial participants and their sexual partners that are likely to be acceptable and appropriate to a range of stakeholders. These strategies include implementing measures during the informed consent process, supporting disclosure of research participation, providing materials and resources, and developing contingencies in case of incident STIs. All types of informants expressed overwhelming support for simple and clear information around HIV transmission risks during ATIs. Most acknowledged the need for consideration of context-specific aspects surrounding HIV disclosure. Similarly, most interviewees reported that research teams should not mandate disclosure of ATI research participation and sensitivity should be given to relationship and partnership dynamics, as well as underlying social and psychological issues. Nevertheless, informants appreciated the complexity of non-ATI disclosure in cases where HIV transmission risk may be possible or imminent.

Our study yielded rich considerations for risk mitigation measures that included regular HIV/STI counseling, sustained dialogue, mental health support, case-by-case evaluations, and $\mathrm{CAB}$ involvement in determining appropriate measures. Informants suggested possible ways to simplify information about ATIs and partner protection measures, including companion documents to improve the quality of informed consent, videos, presentations, fact sheets, websites, visual language, workshops, and role plays. In cases of incident STIs, most informants agreed on the need for treatment, counseling, and contact tracing, although there was no consensus on when or whether to disqualify ATI trial participants presenting with STIs. Some informants believed it would be too punitive to limit ATI trial participation due to incident STIs, but most agreed repeat STIs should require more careful caseby-case attention. Informants highlighted the critical role of community consultation in determining how to deal with incident STIs in the context of ATI trials.

Our study points to the need for the design of possible risk mitigation toolkits for ATIs. One such proposed toolkit is comprised of HIV/ATI disclosure checklists and standardized counseling guides around transmission risks prior and during ATIs. ${ }^{3}$ Our data revealed that allowing ATI participants to become and stay viremic, equivalent to breaking the $\mathrm{U}=\mathrm{U}$ equation, re-introduces the need for complex negotiations and decision-making related to HIV and research disclosure, safe sex negotiations, and partner protection. In fact, prior to PrEP and $\mathrm{U}=\mathrm{U}, \mathrm{PLWH}$ had to navigate these same risks and strategies. ${ }^{18}$

In terms of measures for protection of sexual partners of ATI trial participants, data from all informants indicated a priority for PrEP referral and/or provision for partners, but highlighted important nuances as well. Some of these considerations included the need to appreciate the full HIV prevention toolkit of effective technologies (e.g., vaginal rings, condoms, etc.) for sexual partners. Scholars have advised doing more research on PrEP efficacy in the context sudden rebounds of viremia. ${ }^{11,19}$ While informants stressed the need to think "beyond PrEP" for partner protection, there was no mention of the role post-exposure prophylaxis (PEP) may play in mitigating HIV transmission risks during ATIs. ${ }^{2}$

PrEP and PEP referral and/or provision may prove more challenging in situations where ATI trial participants have multiple and/or anonymous partners, particularly if the partners do not have medical insurance. Research teams need to appreciate that PrEP and PEP availability, acceptability, and/or uptake remain lower in communities of color, ${ }^{20}$ as well as societal and structural-level barriers to PrEP and PEP access. ${ }^{21}$ Further, there was no discusssion about the legal responsibility that would follow investigators who directly prescribe PrEP and PEP to partners. More deliberative work will be necessary to determine whether it will be feasible for HIV cure research teams and/or large research networks to provide (or refer for) partner protection measures, such as PrEP/PEP. Research teams and/or networks may consider preparing partner protection measure guidelines with community, ethics and legal input before initiating ATI trials (e.g., plans and/or standard operating procedures), and updating them as needed. Many of the issues highlighted in this manuscript could be solved by larger efforts to ensure PrEP is widely available and accessible to all individuals regardless of insurance status or ability to pay.

Our findings add to normative guidance on possible risk mitigation strategies for ATI non-participants. ${ }^{6,7}$ For example, Eyal proposed a "low-hanging fruit" approach to ATI risk mitigation with protections implemented for participants and partners across the arc of ATI trials (e.g., recruitment, informed consent, ATI and ART restart). ${ }^{13}$ In addition, Eyal proposed 
providing HIV treatment support to partners in the event of HIV acquisition. ${ }^{13}$ However, no consensus has yet been achieved as to whether sexual partners who acquire HIV during ATI trials are owed HIV treatment. Nevertheless, this topic expands debates around standards of care owed to HIV prevention trial participants who acquire HIV in the course of their trial participation. ${ }^{22}$ Dawson favors a relational ethics approach to partner protection and argues research teams should at least assume some responsibility for mitigating risks to partners of ATI trial participants because trial procedures create additional HIV transmission and acquisition risks. ${ }^{8}$ Dawson summarized four layers of responsibility in the context of ATI trials: 1) researchers' obligations to trial participants, 2) researchers' obligations to partners, 3) participants' responsibilities to partners, and 4) partners' protection to practice safe sex in general. ${ }^{8}$

The conundrum of what should be included in risk mitigation packages for ATI trial participants parallels similar long-standing debates in the HIV prevention field around standards of prevention. ${ }^{23-27}$ With the availability of safe and effective biomedical forms of prevention (e.g., oral PrEP), the contents of the standards of prevention packages for HIV prevention trials have evolved over time. ${ }^{23}$ In HIV prevention trials, the standard historically includes HIV testing, counseling, condoms, and sometimes STI testing and treatment. ${ }^{25}$ The arrival of efficacious, long-acting injectable PrEP and other emerging methods such as the vaginal ring is likely to further shift this standard of prevention. ${ }^{25,28}$ Further, distinctions may need to be made between ethical obligations (basic requirements that must be met) versus ethical aspirations (desirable but not required). ${ }^{26}$ Importantly, as highlighted by our informants, determining minimal risk mitigation packages for sexual partners of ATI trial participants will require robust and sustained community and stakeholder engagement to ensure appropriateness, acceptability, and sensitivity to local contexts and cultural issues. ${ }^{24}$ Risk mitigation packages will need to be adapted to local settings and norms as well as resources, such as PrEP and HIV prevention availability.

Our summary of possible ethical and practical considerations for mitigating risks to sexual partners from ATIs derived from our study can be found in Table 3. This list is not exhaustive and may not reflect the views of all stakeholders involved in HIV cure-related research. Some of these considerations may apply to situations outside of ATI trials (e.g., virally suppressed PLWH who decide to discontinue ART or PLWH unable to achieve an undetectable status).

\section{Limitations}

The results of this study should be interpreted in light of its limitations. The purposive nature of the interviews, together with informants self-selection following a formal invitation, may have introduced a sampling bias. Funding constraints prevented us from interviewing additional informants, including more HIV clinicians, especially community clincians. The ongoing COVID-19 pandemic precluded conducting interviews with additional HIV/infectious diseases doctors and clinicians at the time we conducted interviews due to their clinical responsibilities. This important gap will need to be investigated in a follow-up project. Another limitation is that we did not interview sexual partners of ATI trial participants as part of this study. To the best of our knowledge, no PLWH interviewed previously participated in an ATI trial. We did not examine specifically the potential effects of ATIs on behavioral inhibitions, sexual desires, sexual abstinence or in the context of substance use during sexual activity, also known as chemsex. After carefully reviewing all interview transcripts, two interviewers and data coders (K.D. and J.K.) independently determined that saturation had not been reached; i.e., the point when no new information emerges. ${ }^{29}$ New information and considerations continued to emerge during each interview, especially in regard to different respondent groups, but project resources precluded conducting additional interviews. Nevertheless, we identified a series of important findings that are of relevance to the ethical implementation of ATIs. In addition, as in much qualitative research, our findings should be viewed as hypothesis generating, which will be relevant for future data gathering efforts. Our findings may also have been enriched by incorporating the views of former and current ATI trial participants and their sexual partners, including those in HIV sero-different relationships, despite that it might represent a retrospective, rather than prospective assessment of the issues at hand. Nonetheless, the topic of risk mitigation to sexual partners during ATIs will require broad stakeholder input. Considerations were also likely skewed towards the U.S. context, since all our informants were affiliated with U.S.-based institutions. Our research was not designed as a consensus study; therefore, additional work will be required to generate consensus on the proposed ethical and practical partner protection measures during ATIs in various contexts including ethical obligations versus aspirations. Research methods could also be enriched by dyadic approaches involving both ATI trial participants and their partners. 
Table 3. Summary of possible ethical and practical considerations for mitigating risks to sexual partners during ATls in HIV cure-related research (United States, 2020).

Considerations for ATI Participants

- Informed consent information around risk of HIV transmission to sexual partners during ATIs should remain as clear as possible. Different modalities can be employed for different communities, including women and racial and ethnic minorities to increase participant understanding of informed consent materials such as companion pieces and consent videos. An assessment of understanding is recommended. There should be adequate community involvement around the design of informed consent strategies.

- Disclosure of HIV status to sexual partners should be encouraged, but not be mandated or enforced. Attention should be paid to cultural aspects and relationship dynamics around HIV disclosure. Research teams should support ATI trial participants, through counseling, the provision of negotiation skills and/or disclosure scripts, etc., should they wish to disclose.

- Disclosure of ATI research participation to sexual partners should be encouraged but not be required. However, research teams should support participants through the disclosure process, particularly in cases of HIV sero-different relationships, unprotected sex with HIV-negative partners, and when $U=U$ is relied upon as the HIV prevention method. There should be sensitivity to relationship dynamics, as well as social, psychological, and cultural issues around disclosure.

- In complex situations where there is no ATI disclosure and risk of onward transmission, there should be counseling on risks of HIV/STI transmission to partners, dialogue, education and mental health support. Situations should be reviewed on a case-by-case basis and attention paid to complex relationships dynamics and social/sexual networks. Participants should be made aware of local laws around HIV/STI transmission.

- Research teams should consider developing materials to simplify ATI information and clearly explain the need for partner protection. These can include documents to accompany the informed consent process, videos, presentations, fact sheets, websites, and role plays, among others that are appropriate for all affected communities. Materials should help empower participants around possible trade-offs and could also be used with HIV care providers or in community engagement efforts.

- In cases of incident STIs during ATI trials, participants should be referred for prompt treatment, counseling, and contact tracing.

- Research teams must carefully consider whether discontinuing participants would be too punitive and engage communities to determine the best way to deal with incident STIs in different contexts. More consensus should be generated in the community on how to deal with incident STls during ATI trials.

Considerations for Sexual Partners of ATI Study Participants

- To the extent possible, HIV-negative sexual partners of ATI study participants should be referred to receive PrEP. More deliberative work will be needed to define the role of daily PrEP versus on-demand PrEP in the context of ATI trials. In addition to PrEP, other HIV prevention options should be discussed, such as external or internal condoms, dapivirine vaginal rings for female partners, voluntary medical male circumcision, etc.

- There should be more in-depth discussion and careful navigation in situations involving multiple and anonymous partners. More caution should be exercised when ATI trial participants disclose having multiple and/or anonymous sexual partners. More consensus should be generated in the community on how to deal with multiple and/or anonymous sexual partners during ATIs.

- Careful attention should also be paid to socio-cultural dynamics where PrEP availability, accessibility, acceptability, and/or uptake may be lower.

- Partners should be invited to come to the research sites to receive additional counseling if needed, although this should not be mandatory. Research teams can explore innovative ways to engage partners, such as telehealth visits.

- Robust standards of risk mitigation measures should be in place with all partners. Research teams should acknowledge people's lived experiences and foster prevention altruism ${ }^{31}$ among ATI trial participants. All parties involved should recognize unintended secondary HIV transmission events may considerably set back the field of HIV cure-related research.

- Research teams should consider developing materials for partners of ATI trial participants that explain possible risks of the study and mitigation strategies which are appropriate for all affected communities. These could include slides, frequently asked questions, videos, flowcharts, letters, vignettes, scenarios, scripts, handouts, etc.

- Ultimately, partner protection should be about building relationships, trust, and good communication. Research teams and/or networks may consider developing partner protection guidelines before initiating ATI trials (e.g., plans and/or standard operating procedures) and updating them as needed.

\section{Conclusions}

Prevention of HIV transmission to sexual partners during ATIs remains one of the most pressing ethical issues surrounding HIV cure-related trials. ${ }^{6,21}$ Because ATIs go against standards of clinical care for trial participants, robust risk mitigation packages are needed for them and their sexual partners. While there is no way to completely eliminate the risk of HIV transmission during ATIs, HIV cure trialists and sponsors need consider the ethical issues surrounding sexual partners of ATI participants and thereby enhance the trustworthiness of the the research. ${ }^{13,30}$

\section{List of Abbreviations}

ACTG AIDS Clinical Trials Group

ART Antiretroviral Treatment

ATI Analytical Treatment Interruption
AVRC AntiViral Research Center

CAB Community Advisory Board

CAPS Center for AIDS Prevention studies

CBO Community-Based Organization

DARE Delaney AIDS Research Enterprise

HARP-PS HIV + Aging Research Project -

Palm Springs

HIPAA Health Insurance Portability and

Accountability Act

IRB Institutional Review Board

LGBTQ+ Lesbian, Gay, Bisexual, Transgender,

Queer +

NGO Non-Governmental Organization

PEP Post-Exposure Prophylaxis

PLWH People Living with HIV

PrEP Pre-Exposure Prophylaxis

STI Sexually Transmitted Infection 
UNC-CH University of North Carolina at Chapel Hill

U.S. United States

$\mathrm{U}=\mathrm{U} \quad$ Undetectable $=$ Untransmittable

UCSF University of California San Francisco

\section{Acknowledgements}

The authors thank the amfAR Institute for HIV Cure Research CAB, the AIDS Clinical Trials Group (ACTG) Community Scientific Sub-Committee, the Martin Delaney Collaboratories Towards an HIV-1 Cure CABs - in particular the Delaney AIDS Research Enterprise (DARE) CAB.

\section{Availability of data and material}

All relevant quotes have been included in the results section.

\section{Author's contributions}

KD drafted the initial version of this manuscript.

J.K., L.D., J.T., D.M.C., B.B., M.O.J., P.S., J.A.S., J.S., and M.J.P. reviewed the manuscript for intellectual contents.

All authors read and approved the final manuscript.

\section{Consent for publication}

Participants provided informed consent to publish deidentified data.

\section{Competing interest}

Jeremy Sugarman is a member of Merck KGaA's the Bioethics Advisory Panel Stem Cell Research Oversight Committee; is a member of IQVIA's Ethics Advisory Panel; is a member of Aspen Neurosciences Scientific Advisory Board; is a consultant to Biogen; and has consulted for Portola Pharmaceuticals, Inc. These are unrelated to the material discussed in this manuscript. The other authors declare that they have no competing interests.

\section{Ethics approval and consent to participate}

The Institutional Review Board of the University of North Carolina at Chapel Hill approved this empirical research ethics study (study \#: 19-0522). All interview participants included in this study provided informed consent.

\section{Funding}

This work was supported by an Ethics Administrative Supplement to R21MH118120 to K.D. In addition, M.J.P., J.A.S. and K.D. are grateful for support received from the amfAR Institute for HIV Cure
Research (amfAR 109301). K.D. received support from UM1AI126620 (BEAT-HIV Collaboratory) cofunded by NIAID, NIMH, NINDS and NIDA. P.S. and K.D. received support from R21MH122280. MJP received funding from a training grant NIH/NIAID T32 AI60530-12 and through the UCSF Resource Allocation Program.

\section{ORCID}

Karine Dubé (D) http://orcid.org/0000-0003-3458-1539 Brandon Brown (D) http://orcid.org/0000-0002-

6348-4108

\section{References}

1 Prevention Access Campaign. U =U. https://www.preventionaccess.org/

2 Julg B, Dee L, Ananworanich J, et al. Recommendations for analytical antiretroviral treatment interruptions in HIV research trials-report of a consensus meeting. Lancet Hiv. 2019;6(4): e259-e268.

3 Peluso MJ, Dee L, Campbell D, et al. Collaborative, multidisciplinary approach to HIV transmission risk mitigation during analytic treatment interruption. J Virus Erad. 2020;6(1):34-37.

4 Hardy DW. Analytical treatment interruptions and human immunodeficiency virus cure research: seizing the opportunity while maintaining safety and respect. Clin Infect Dis. 2020; 70(7):1418-1420.

5 Sivay M, Palumbo P, Zhang Y, et al. HIV drug resistance, phylogenetic analysis, and superinfection among men who have sex with men and transgender women in Sub-Saharan Africa: HPTN 075. Clin Infect Dis. 2020.

6 Eyal N, Deeks SG. Risk to nonparticipants in HIV remission studies with treatment interruption: a symposium. J Infect Dis. 2019;220:1-4.

7 Eyal N, Lipsitch M, Bärnighausen T, Wikler D. Opinion: risk to study nonparticipants: a procedural approach. Proc Natl Acad Sci USA. 2018;115(32):8051-8053.

8 Dawson L. Human immunodeficiency virus transmission risk in analytical treatment interruption studies: relational factors and moral responsibility. J Infect Dis. 2019;220(Suppl 1):S12-S15.

9 Lelièvre JD, Hocqueloux L. Unintended HIV-1 transmission to a sex partner in a study of a therapeutic vaccine candidate. $J$ Infect Dis. 2019;

10 Palich R, Ghosn J, Chaillon A, et al. Viral rebound in semen after antiretroviral treatment interruption in an HIV therapeutic vaccine double-blind trial. Aids. 2019;33(2):279-284.

11 Lelièvre J-D. Preexposure prophylaxis for mitigating risk of HIV transmission during HIV cure - related clinical trials with a treatment interruption. J Infect Dis. 2019;12:1-3.

12 Ugarte A, Romero Y, Tricas A, Casado C, Garcia F, Leal L. Unintended HIV-1 infection during analytical treatment interruption. J Infect Dis. 2019.

13 Eyal N. How to address the risk of HIV transmission in remission studies with treatment interruption: the low-hanging fruit approach. J Infect Dis. 2019;220(Suppl 1):S7-S11.

14 Eyal N, Magalhaes M. Is it ethical to isolate study participants to prevent HIV transmission during trials with an analytical treatment interruption? J Infect Dis. 2019;220(Suppl 1):S19-S21.

15 Eyal N. Removing one barrier to protecting sex partners in HIV remission studies with a treatment interruption. J Infect Dis. 2019;220(Suppl 1):S22-S3.

16 TAG. Research toward a cure trials [Internet]. 2019. http://www. treatmentactiongroup.org/cure/trials

17 Cresswell J. Research Design. Qualitative, Quantitative, and Mixed Methods Approaches. 4th ed. Thousand Oaks, CA: SAGE Publications; 2013.

18 Grace D, Chown SA, Kwag M, Steinberg M, Lim E, Gilbert M. Becoming "undetectable": longitudinal narratives of gay men's 
sex lives after a recent HIV diagnosis. AIDS Educ Prev. 2015; 27(4):333-349.

19 Garner SA, Rennie S, Ananworanich J, Dubé K, et al. Interrupting antiretroviral treatment in HIV cure research: scientific and ethical considerations. J Virus Erad. 2017;3(2):82-84.

20 Sullivan PS, Mena L, Elopre L, Siegler AJ. Implementation strategies to increase PrEP uptake in the South. Curr HIV/AIDS Rep. 2019;16(4):259-269.

21 Dee L, Boone CA, Palm D, Campbell D, Dubé K. Secondary HIV infection and mitigation in cure-related HIV trials during analytical treatment interruptions. J Infect Dis. 2019;220:34-37.

22 UNAIDS. Ethical considerations in biomedical HIV prevention trials [Internet]. 2012. https://www.unaids.org/en/resources/documents/2012/20120701_jc1399_ethical_considerations

23 Haire B, Folayan MO, Hankins C, et al. Ethical considerations in determining standard of prevention packages for HIV prevention trials: examining PrEP. Dev World Bioethic. 2013;13(2): 87-94.

24 Sugarman J, Celum C, Donnell D, Mayer K. Ethical considerations for new HIV prevention trials. Lancet. 2019;6(8):e48991-e491.
25 Sugarman J. Ethical considerations regarding oral preexposure prophylaxis in HIV prevention trials. Curr Opin HIV Aids. 2016; 11(1):109-115.

26 Brown BJ, Sugarman J. Why ethics guidance needs to be updated for contemporary HIV prevention research. J Int AIDS Soc. 2020;23(5):e25500

27 Dawson L, Zwerski S. Clinical trial design for HIV prevention research: determining standards of prevention. Bioethics. 2015; 29(5):316-323.

28 Kanazawa JT, Saberi P, Sauceda JA, Dubé K. The LAIs are coming! Implementation science considerations for long-acting injectable antiretroviral therapy in the United States: a scoping review. AIDS Res Hum Retroviruses. 2021;37(2):75-88.

29 Guest G, Bunce A, Johnson L. How many interviews are enough?: An experiment with data saturation and variability. Field Methods. 2006;18(1):59-82.

30 Eyal N, Kuritzkes DR. Challenges in clinical trial design for HIV-1 cure research. Lancet. 2013;382(9903):1464-1465.

31 O'Dell BL, Rosser BRS, Miner MH, Jacoby SM. HIV prevention altruism and sexual risk behavior in HIV-positive men who have sex with men. AIDS Behav. 2008;12(5):713-720. 